\title{
Investigating the Response to Dofetilide with Symmetric Projection Attractor Reconstruction of the Electrocardiogram
}

\author{
Jane V Lyle ${ }^{1}$, Manasi Nandi ${ }^{2}$, Philip J Aston ${ }^{1}$ \\ ${ }^{1}$ University of Surrey, Guildford, UK \\ ${ }^{2}$ King's College London, London, UK
}

\begin{abstract}
The aim of this study is a preliminary investigation into the application of our novel Symmetric Projection Attractor Reconstruction (SPAR) method to the electrocardiogram (ECG) signals of individuals treated with the cardioactive drug dofetilide. We show that our SPAR technique correlates with standard assessment, and is also able to discriminate gender from the ECG response to dofetilide more accurately than the standard metrics.
\end{abstract}

\section{Introduction}

Dofetilide is an antiarrhythmic medication prescribed to patients with atrial fibrillation and flutter [1]. However, its effect on specific potassium channels may prolong repolarisation, increasing the risk of the potentially fatal torsades de pointes [2]. Furthermore, dofetilide is more frequently associated with torsades de pointes or a need to discontinue in females than males, although this is not reflected in standard prescribing guidelines and the complete biological mechanisms underlying this are not clear [3].

The electrocardiogram (ECG) is a key tool for monitoring patients taking dofetilide, with the identification of particular points on the ECG wave [4], being the mainstay of analysis. However, such techniques excludes the wealth of information in the waveform profile. Our Symmetric Projection Attractor Reconstruction (SPAR) method is a novel approach that generates a two-dimensional image from all of the data, facilitating quantification of different aspects of ECG morphology. SPAR analysis has been applied to various approximately periodic cardiovascular signals [5-7], where it has been shown to supplement standard assessment. We have also previously demonstrated that the SPAR method can discriminate gender in young healthy adults more successfully than existing measures [8].

This study provides a preliminary investigation of the nature of the SPAR images in response to the administration of dofetilide, and compares this with the changes observed in standard ECG metrics.

\section{The SPAR method}

Symmetric Projection Attractor Reconstruction (SPAR) aims to provide a means of visualising and quantifying the morphology of any approximately periodic signal. The complexity of the ECG and the difficulty in identifying specific points accurately and without bias make ECG an ideal signal for analysis with this technique. The SPAR method using 3 points has been described previously [7], and is extended to a higher number of points in [9].

For an ECG time series $x(t)$ we apply the approach of Takens' delay coordinate embedding [10]. We place $n \geq 3$ points at equal distances of $\tau$ apart on the signal, where $\tau$ is chosen to be $1 / n$ of the average cycle length (cardiac cycle duration) of the ECG. Thus $n$ points generate $n$ time series as $x_{i}(t)=x(t-(i-1) \tau)$ for $i=1, \ldots, n$ to give us an object bounded in $n$-dimensional phase space. To obtain an output that is both visually informative and where change is easily quantified, we then generate a two-dimensional projection $(v, w)$ that has symmetric properties. We add a density to provide information about areas visited more or less frequently, and call this final image our 'attractor'.

Figure 1 illustrates an embedding with $n=3$ points. The attractor of a normal Lead II signal, as shown in Fig. 1(iv), has three long arms, each corresponding to the traverse of a given $\mathrm{R}$ peak by one of the embedded points $x_{1}$, $x_{2}$ or $x_{3}$. The higher densities seen on these arms near the centre are a result of the $\mathrm{T}$ wave, and the shape of the arms is largely determined by the duration of the QT interval.

\section{Methods}

\subsection{Clinical dataset and data processing}

Records for 22 individuals (19 to 35 years, 11 female) were taken from the Physionet ecgrdvq database [11]. The original study [2] was a five treatment crossover trial, but we only required the 2,112 Lead II ECG recordings associated with placebo and dofetilide. Baseline ECG recordings were taken prior to treatment, and 15 subsequent triplicate recordings were made at fixed timepoints over 24 hours. 

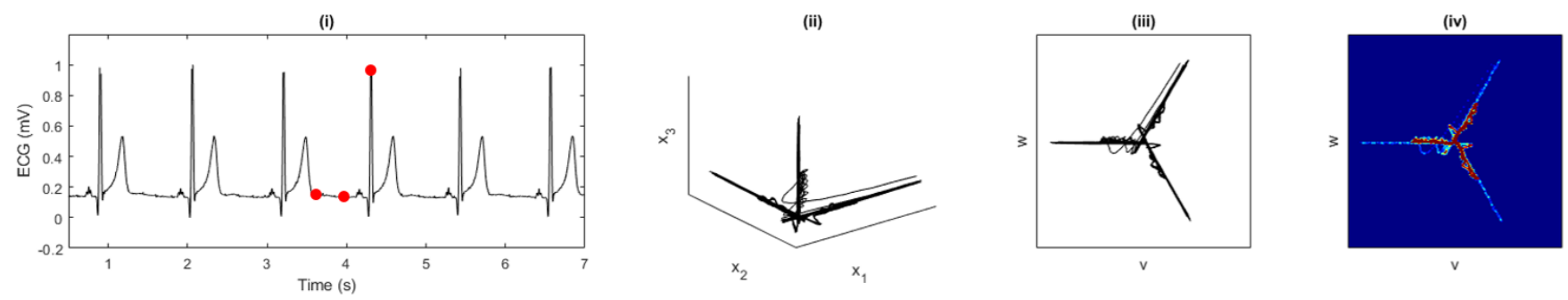

Figure 1. SPAR for an ECG signal embedded with $n=3$ points. From left to right: (i) ECG with $n=3$ points generating time series $x_{1}, x_{2}, x_{3}$, (ii) Object in three-dimensional phase space, (iii) Two-dimensional projection of the three-dimensional object with three-fold symmetry, (iv) Attractor with density. Illustration adapted from [8] Figure 1.

The ECG signals were short and of good quality. Data was normalised to give an $\mathrm{R}$ peak height of 1 from the isoelectric line. No additional filtering or data selection was applied in order to demonstrate that SPAR is a robust method. The SPAR method was used to embed the ECGs with $n=3, \ldots, 8$ points, generating 12 two-dimensional attractors. We extracted two measures from each attractor to quantify the density distribution of each projection.

To provide a comparison to a standard approach to ECG analysis, we took the measures provided with the original study; six interval measures RR, PR, QRS, QTc, J-T peak $\mathrm{c}$, $\mathrm{T}_{\text {peak }}-\mathrm{T}_{\text {end }}$ (taking QTc by Fridericia's correction and J$\mathrm{T}_{\text {peak }} \mathrm{c}$ as $\mathrm{J}-\mathrm{T}_{\text {peak }} / \mathrm{RR}^{0.58}$ ), and five repolarisation measures; early and late repolarisation durations, $\mathrm{T}$ wave amplitude, $\mathrm{T}$ wave symmetry and flatness [12].

Assessing the impact of dofetilide requires the change from baseline to be considered. Therefore, we derived a new set of metrics by taking the difference of all absolute measures from the individual's average baseline values, and then applying placebo-correction by timepoint. This new set of metrics is denoted by $\triangle \triangle$.

\subsection{Machine learning: SVM}

Our previous study [8] showed that the SPAR method provides high accuracy in discriminating gender from the ECG. We therefore considered whether a gender difference could be distinguished from the changes seen after dosing with dofetilide. A linear support vector machine (SVM) was applied [13], taking each individual as the validation set in turn, giving an average cross-validated classification accuracy. Four different feature groupings were taken from the $\triangle \triangle$ metrics: Intervals, Intervals + Repolarisation, Attractor, All of above. Feature reduction was achieved through a forward feature selection algorithm [14] over 20 runs per feature group, and optimising for the feature set providing the highest accuracy.

\section{Results}

\subsection{Response to dofetilide}

The original study showed that dofetilide prolonged the QTc interval, with equal prolongation of its $\mathrm{J}_{-} \mathrm{T}_{\text {peak }} \mathrm{c}$ and $\mathrm{T}_{\text {peak }}-\mathrm{T}_{\text {end }}$ components [2]. Since the shape of the attractor arms reflects the QT duration, it would be expected that changes in the attractor are closely correlated to changes in QT. This is indeed the case, and the mean 3 point attractor metric $\triangle \triangle r_{3}$ has a high correlation of 0.95 with the mean $\triangle \triangle \mathrm{QTc}$ by timepoint over the first 8 hours, and a correlation of 0.91 over 24 hours. Figure 2 illustrates this.

\subsection{Higher $n$ embedding}

Previous SPAR studies [5-8] have utilised an $n=3$ point embedding. However, the complexity of ECG merits an embedding in more points to aid the discrimination of certain features. The optimum embedding is dependent on both the problem and the individual, hence the use of attractors embedded with $n=3, \ldots, 8$ points for this study.

Figure 3 illustrates that generating attractors with multiple points concurrently provides more information about the complex ECG signal. The ECG signals for an individual at baseline and 2.5 hours post-dose clearly show a QT prolongation and a reduced $\mathrm{T}$ wave amplitude. It can be shown, for example, that the combination of information from the 3 point attractor and 5 point attractor indicate that the QT prolongation is accompanied by a change in the $\mathrm{T}$ wave morphology.

\subsection{Gender basis to response}

We applied a linear SVM to the $\triangle \triangle$ metrics from the attractor and the original study, considering the trajectory of change in the period from treatment to each of four timepoints, as shown in Table 1.

It was possible to distinguish gender correctly from the 


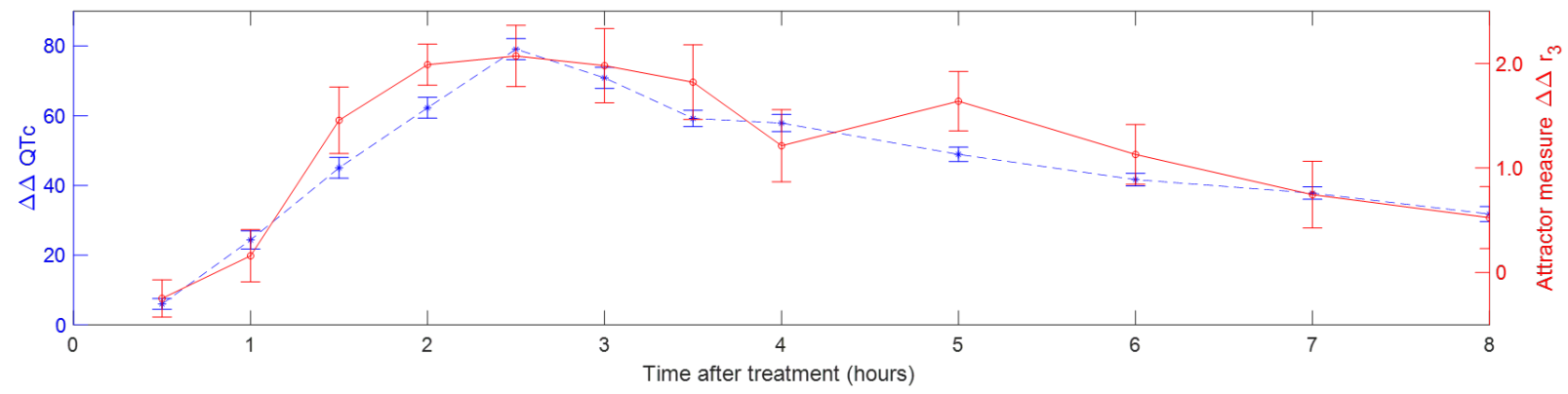

Figure 2. A high correlation is observed between the mean $\triangle \triangle \mathrm{QTc}$ (blue) and attractor $\triangle \triangle \mathrm{r}_{3}$ (red) metrics over 8 hours after treatment (bars indicate the standard error for each metric at the timepoint.)
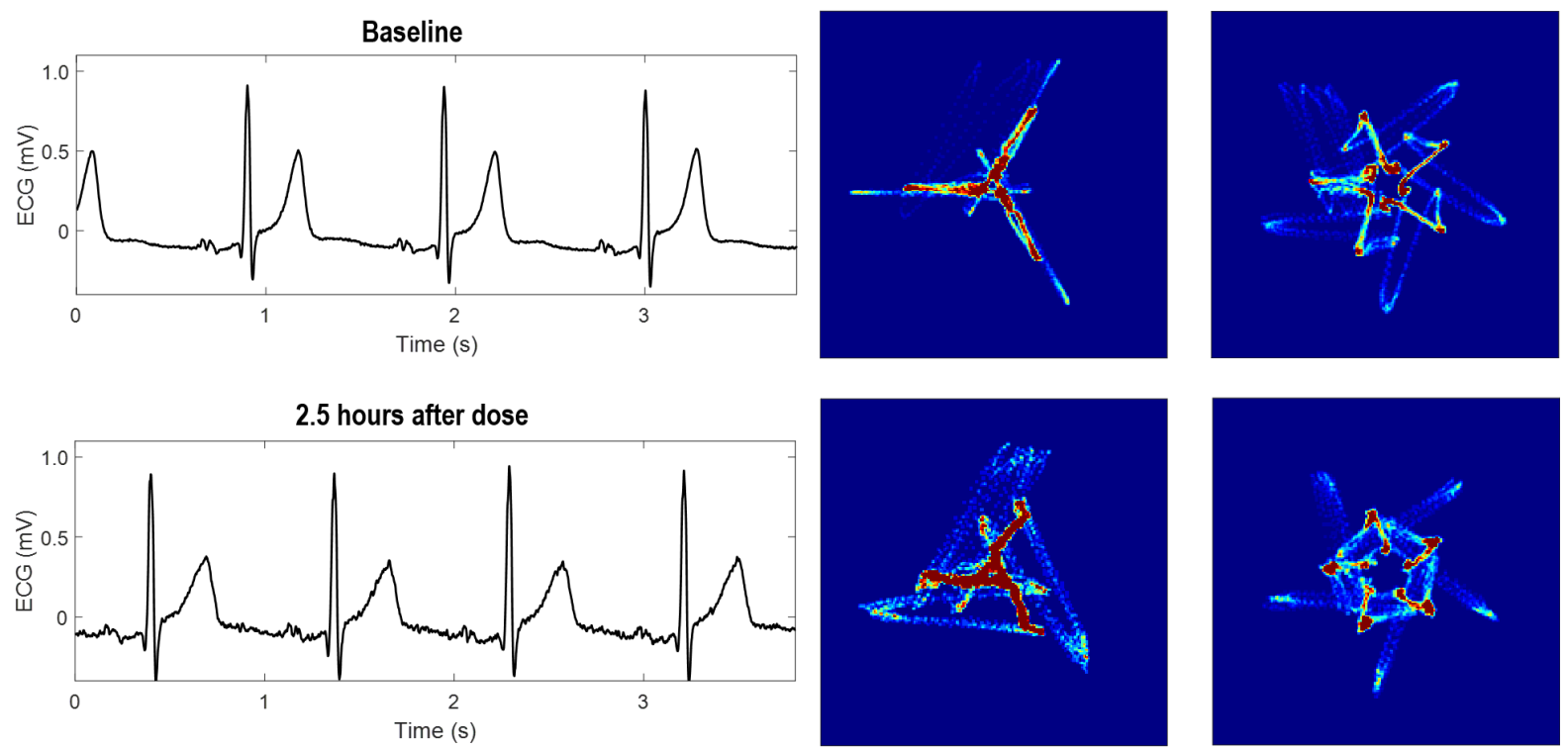

Figure 3. An individual's ECG signals at baseline and 2.5 hours, with 3 (centre) and 5 (right) point embedded attractors.

change in the first 0.5 hour post-dose in at least 18 of the 22 individuals. The best result of $95.5 \%(21 / 22)$ was achieved with a combination of SPAR, interval and repolarisation features. By 2.5 hours (the mean time for peak serum concentration of dofetilide), $100.0 \%$ accuracy was observed for the combined features, although the SPAR features alone $(95.5 \%)$ performed better than the interval or repolarisation features $(81.8 \%)$. For the trajectory over 4 hours, an accuracy of $100.0 \%$ was achieved with only 4 attractor metrics. This $100.0 \%$ accuracy continued for SPAR features over the 24 hour period, whereas accuracy for interval and repolarisation features fell to $72.7 \%$ (16/22).

We then considered the various features selected in the classification. Interestingly, for interval measures, changes in the PR interval were key for timepoints up to 4 hours. We would not expect the PR interval to change in response to dofetilide [1], but the specific question of gender discrimination from such data has not been previously considered; further work on this is required. The $\mathrm{T}$ wave amplitude, flatness and asymmetry metrics were included when the repolarisation features were added. SPAR features utilised in the classification were spread across all embeddings. At 24 hours, $100.0 \%$ classification could be achieved with measures from only 4, 6 and 7 point embeddings. Throughout, misclassification impacted both male and female records approximately equally.

\section{Discussion and Conclusions}

In this study we have demonstrated the application of multi-point attractors generated by the SPAR method to the analysis of ECG signals following treatment with dofetilide. We observed that the ECG attractor provides metrics that correlate to changes in the QTc interval, and allows us to discriminate a difference in gender response.

The determination of the interval and repolarisation measures in the original study required considerable processing and human verification $[2,12]$. In comparison, the SPAR method is much simpler to apply. The original study also utilised multiple leads of the ECG, whereas we only 
Table 1. Cross-validated accuracy for gender classification from the trajectory of $\triangle \Delta$ metrics at four timepoints. The minimum number of features required from each grouping to achieve the result is indicated by $(n)$.

\begin{tabular}{lcccc}
\hline \hline & $\mathbf{0 . 5 h}$ & $\mathbf{2 . 5 h}$ & $\mathbf{4 h}$ & $\mathbf{2 4 h}$ \\
\hline Interval & $81.8 \%$ & $81.8 \%$ & $77.3 \%$ & $72.7 \%$ \\
6 features & $(1)$ & $(6)$ & $(3)$ & $(1)$ \\
Int+Repol & $86.4 \%$ & $81.8 \%$ & $81.8 \%$ & $72.7 \%$ \\
11 features & $(4)$ & $(2)$ & $(2)$ & $(1)$ \\
SPAR & $90.9 \%$ & $95.5 \%$ & $100.0 \%$ & $100.0 \%$ \\
24 features & $(15)$ & $(7)$ & $(4)$ & $(3)$ \\
SPAR+Int+Repol & $95.5 \%$ & $100.0 \%$ & $100.0 \%$ & $100.0 \%$ \\
35 features & $(9)$ & $(9)$ & $(4)$ & $(3)$ \\
\hline \hline
\end{tabular}

used Lead II for the attractor here; extending the SPAR method to the other leads may provide additional information. Furthermore, we used only basic attractor metrics and elementary machine learning to achieve some very promising results. Extending this work to larger datasets may benefit from more complex techniques, and the use of deep learning on the attractor images directly [15].

We only give a simple example here of a correlation between the changes in an attractor metric and changes in the QTc interval. Further investigation could characterise which features reflect subtle changes in the $\mathrm{T}$ wave morphology as a result of dofetilide treatment. We also note that we observe a higher variability in the attractor metrics than the interval and repolarisation metrics. This is a reflection of the individuality over the whole ECG waveform and therefore SPAR may provide more subtlety in quantifying the dofetilide response, especially when more factors, such as age, race and illness, are introduced.

The ability to discriminate gender from the trajectory of change following dofetilide treatment is supported by clinical observation [3]. It is therefore unsurprising that we can make the gender classification reasonably well from the interval and repolarisation metrics. However, the $100.0 \%$ accuracy achieved with SPAR metrics supports that there is additional information that can be obtained by using the whole waveform data. The ability to differentiate gender indicates underlying differences in the action of dofetilide, which could impact on treatment and monitoring.

This preliminary study of the SPAR method has shown that dofetilide causes marked changes to the attractor, and that these changes are better quantified by applying multiple point attractors. A simple gender classification has suggested that SPAR provides information additional to existing ECG analysis, and this may have clinical utility in understanding personalised drug responses and risk.

\section{Acknowledgements}

Jane Lyle is grateful for an EPSRC PhD studentship (EP/M508160/1).

\section{References}

[1] Roukoz, H, Saliba, W. Dofetilide: a New Class III Antiarrhythmic Agent. Exp. Rev. of Cardiovascular Ther. 2007;5(1): 9-19.

[2] Johannesen L, Vicente J et al. Differentiating Drug-Induced Multichannel Block on the Electrocardiogram: Randomized Study of Dofetilide, Quinidine, Ranolazine, and Verapamil. Clin. Pharmacol. Ther. 2014;96(5):549-558.

[3] Pokorney, SD, Yen, DC, Campbell, KB et al. Dofetilide Dose Reductions and Discontinuations in Women Compared with Men. Heart Rhythm. 2018;15(4):478-484.

[4] Clifford GD. ECG statistics. Advanced Methods for ECG Analysis. Artech House; 2006:55-99.

[5] Aston PJ, Nandi M, Christie M, Huang Y. Comparison of Attractor Reconstruction and HRV Methods for Analysing Blood Pressure Data. Comp. Cardio. 2014;41:437-440.

[6] Charlton PH, Camporota L, Smith J, Nandi M, Christie MI, Aston PJ et al. Measurement of Cardiovascular State using Attractor Reconstruction Analysis. Signal Proc. Conf. (EUSIPCO). 2015;444-448.

[7] Aston PJ, Christie M, Huang Y, Nandi M. Beyond HRV: Attractor Reconstruction Using the Entire Cardiovascular Waveform Data. Phys. Meas. 2018;39:024001.

[8] Lyle JV, Charlton PH, Bonet-Luz E, Chaffey G, Christie M, Nandi M, Aston PJ. Beyond HRV: Analysis of ECG Signals Using Attractor Reconstruction. Comp. Cardio. 2017;44.

[9] Lyle JV, Nandi M, Aston PJ. Symmetric Projection Attractor Reconstruction: Higher Embeddings. In preparation.

[10] Takens F. Detecting Strange Attractors in Turbulence. Dynamical Systems and Turbulence, Warwick 1980, Lecture Notes in Mathematics. 1981;898:366-381.

[11] Goldberger AL, Amaral LAN, Glass L et al. PhysioBank, PhysioToolkit, and PhysioNet: Components of a New Research Resource for Complex Physiologic Signals. Circ. 2000;101(23):e215-e220.

[12] Vicente J, Johannesen L et al. Comprehensive T wave Morphology Assessment in a Randomized Clinical Study of Dofetilide, Quinidine, Ranolazine, and Verapamil. J Am Heart Ass. 2015;4:e001615.

[13] Cortes, C, Vapnik, VN. Support-Vector Networks. Machine Learning. 1995;20(3): 273-297.

[14] Guyon, I, Elisseeff, A. An Introduction to Variable and Feature Selection. J. Mach. Learn. Res. 3. 2003;1157-1182.

[15] Aston PJ et al. Deep Learning Applied to Attractor Images Derived from ECG Signals for Detection of Genetic Mutation. Submitted to Comp. Cardio. 2019.

Address for correspondence:

Jane Lyle

Dept. of Mathematics, University of Surrey, GU2 7XH, UK

j.lyle@surrey.ac.uk 